\title{
Ion Emittance Growth Due to Focusing Modulation from Slipping Electron Bunch
}

\author{
G. Wang
}

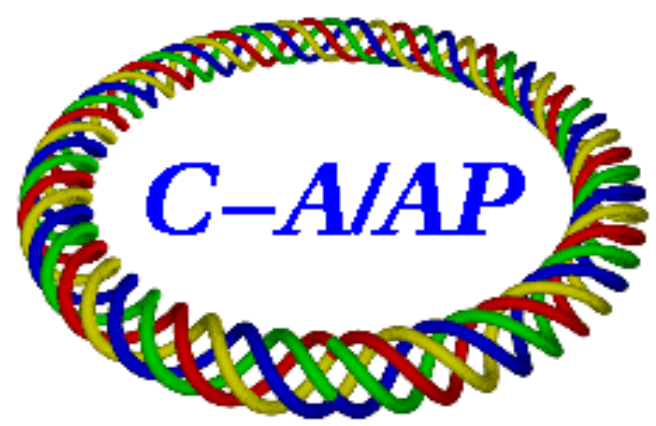

\section{Collider-Accelerator Department Brookhaven National Laboratory Upton, NY 11973}

\section{U.S. Department of Energy Office of Science, Office of Nuclear Physics}

Notice: This document has been authorized by employees of Brookhaven Science Associates, LLC under Contract No. DE-SC0012704 with the U.S. Department of Energy. The United States Government retains a nonexclusive, paid-up, irrevocable, world-wide license to publish or reproduce the published form of this document, or allow others to do so, for United States Government purposes. 


\section{Ion Emittance Growth Due to Focusing Modulation from Slipping Electron Bunch}

Low energy RHIC operation has to be operated at an energy ranging from $\gamma=4.1$ to $\gamma=10$. The energy variation causes the change of revolution frequency. While the rf system for the circulating ion will operate at an exact harmonic of the revolution frequency ( $h=60$ for $4.5 \mathrm{MHz} r f$ and $h=360$ for $28 \mathrm{MHz} r f$.), the superconducting $\mathrm{rf}$ system for the cooling electron beam does not have a frequency tuning range that is wide enough to cover the required changes of revolution frequency. As a result, electron bunches will sit at different locations along the ion bunch from turn to turn, i.e. the slipping of the electron bunch with respect to the circulating ion bunch. At cooling section, ions see a coherent focusing force due to the electrons' space charge, which differs from turn to turn due to the slipping. We will try to estimate how this irregular focusing affects the transverse emittance of the ion bunch.

\section{Time Domain Analysis}

Assuming the line charge distribution of the electrons and the ions are Gaussian, their currents can be written as

$$
I_{e}(t)=\frac{Q_{e}}{\sqrt{2 \pi} \sigma_{e}} \sum_{n=-\infty}^{\infty} \exp \left[-\frac{\left(t-n \cdot \Delta T_{e}\right)^{2}}{2 \sigma_{e}^{2}}\right],
$$

and 1

$$
I_{i}(t)=\frac{Q_{e}}{\sqrt{2 \pi} \sigma_{e}} \sum_{m=-\infty}^{\infty} \exp \left[-\frac{\left(t-m \cdot T_{r e v}\right)^{2}}{2 \sigma_{e}^{2}}\right] .
$$

The electron current as observed by an ion bunch at its $M^{\text {th }}$ revolution is given by

$$
\begin{aligned}
I_{e}(M, \tau) & =\frac{Q_{e}}{\sqrt{2 \pi} \sigma_{e}} \sum_{n=-\infty}^{\infty} \exp \left[-\frac{\left(M \cdot T_{r e v}+\tau-n \cdot \Delta T_{e}\right)^{2}}{2 \sigma_{e}^{2}}\right] \\
& =\frac{Q_{e}}{\sqrt{2 \pi} \sigma_{e}} \sum_{\tilde{n}=-\infty}^{\infty} \exp \left\{-\frac{\left[\tau-\tilde{n} \cdot \Delta T_{e}+\left(M \cdot h_{e}-\operatorname{Round}\left(M \cdot h_{e}\right)\right) \Delta T_{e}\right]^{2}}{2 \sigma_{e}^{2}}\right\}
\end{aligned}
$$

where

$$
h_{e}=\frac{f_{e, r e p}}{f_{\text {rev }}}
$$

with $f_{e, r e p}$ being the repetition frequency of the electron beam and $f_{\text {rev }}$ is RHIC revolution frequency, i.e. $\sim 78 \mathrm{KHz}$. The ions sitting at the center of the bunch, i.e. $\tau=0$, get a kick at its $M^{\text {th }}$ revolution of

\footnotetext{
${ }^{1}$ We consider one circulating ion bunch.
} 


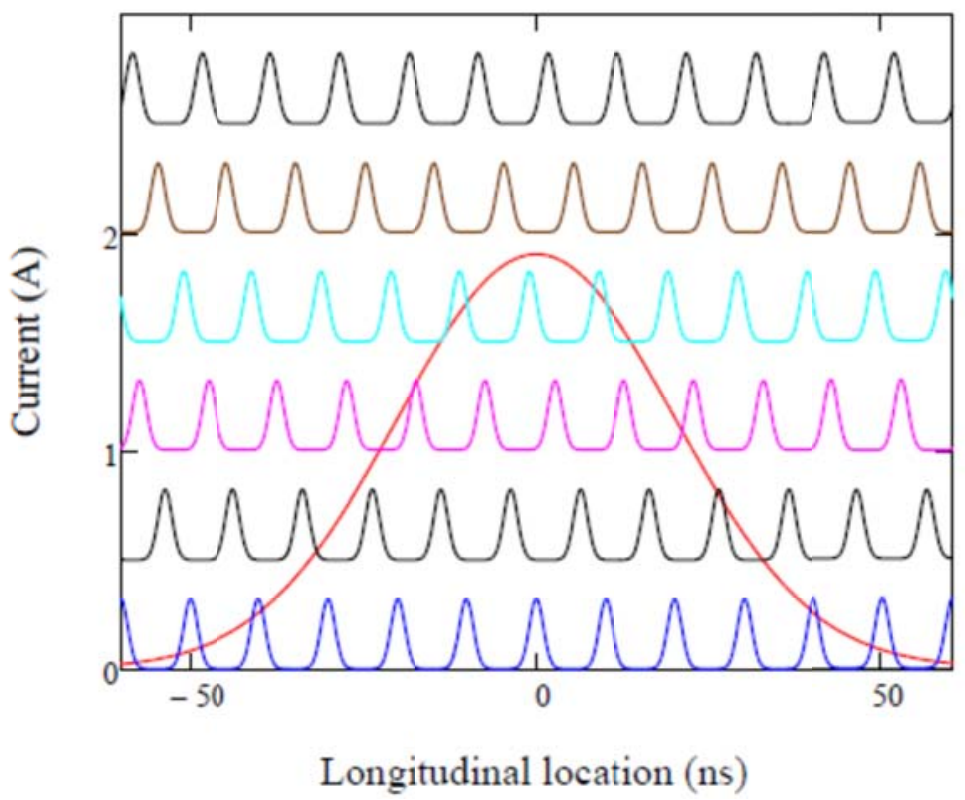

Figure 1: mountain range plot of electron current evolution for 5 successive turns as observed by an ion bunch at the cooling section. The electron current is calculated from eq. (3) for $\gamma_{i}=4.1, \sigma_{e}=1 n s, \Delta T_{e}=10 \mathrm{~ns}, Q_{e}=0.8 n \mathrm{C}, f_{i, f f}=4.56 \mathrm{MHz}$, $\sigma_{i}=5.8 \mathrm{~m} /\left(\beta_{i} c\right)=19.95 \mathrm{~ns}$ and $N_{i}=0.75 \times 10^{9}$.

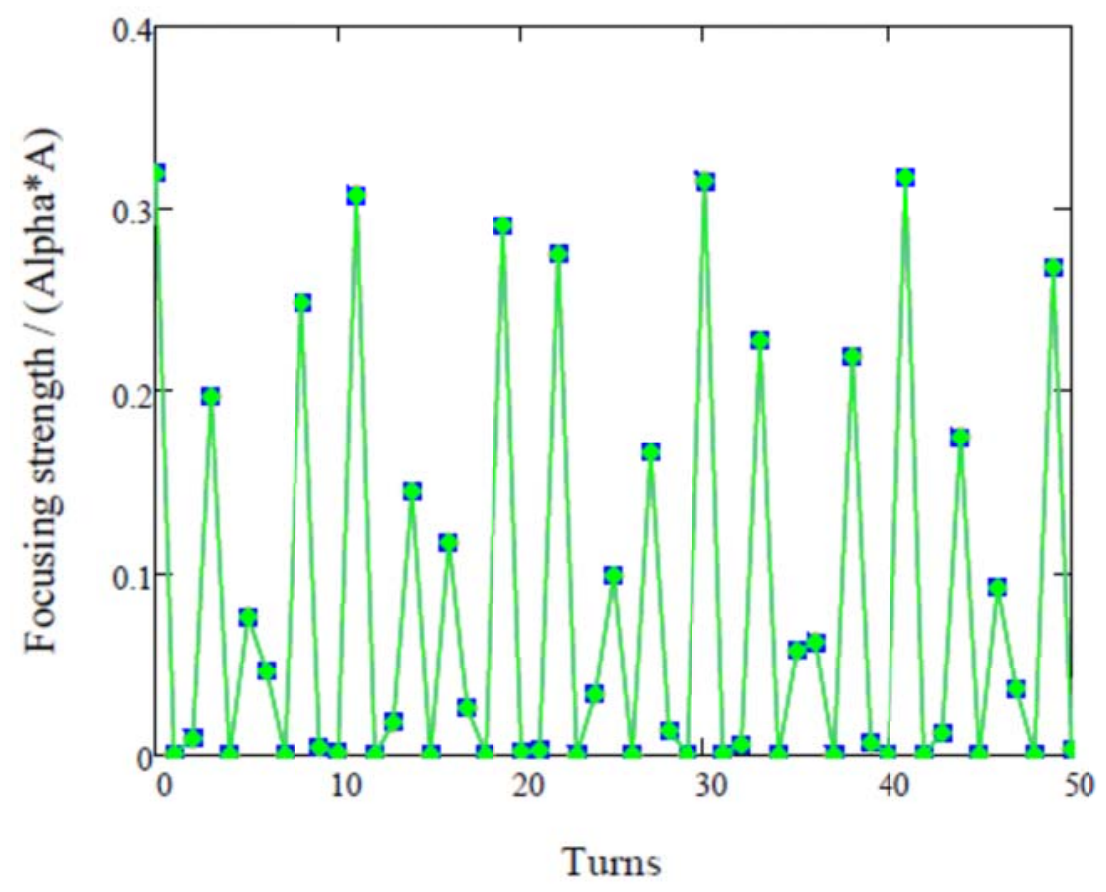

Figure 2: focusing strength seen by ions at the center of the ion bunch in the cooling section. The abscissa is the independent time variable in number of turns and the ordinate is the focusing strength of the electrons at the location of the ion bunch center as calculated from eq. (5) and (8) (they overlap). 


$$
\begin{aligned}
\varepsilon_{M} & =\alpha \cdot I_{e}(M, 0) \\
& =\alpha \cdot \frac{Q_{e}}{\sqrt{2 \pi} \sigma_{e}} \sum_{\tilde{n}=-\infty}^{\infty} \exp \left\{-\frac{\left[-\tilde{n} \cdot \Delta T_{e}+\left(M \cdot h_{e}-\operatorname{Round}\left(M \cdot h_{e}\right)\right) \Delta T_{e}\right]^{2}}{2 \sigma_{e}^{2}}\right\} .
\end{aligned}
$$

Assuming $\Delta T_{e} \gg \sigma_{e}$, only the closest electron bunch, with $\tilde{n}=0$, has contribution and hence eq. (5) simplifies to

$$
\varepsilon_{M}=\alpha \cdot \frac{Q_{e}}{\sqrt{2 \pi} \sigma_{e}} \exp \left\{-\frac{\left[M \cdot h_{e}-\operatorname{Round}\left(M \cdot h_{e}\right)\right]^{2} \Delta T_{e}^{2}}{2 \sigma_{e}^{2}}\right\} .
$$

Splitting $h_{e}$ into the integer part, $h_{e, I}$, and the fractional part, $v_{e}$, i.e.

$$
h_{e}=h_{e, I}+v_{e} \text {, }
$$

eq. (6) becomes

$$
\varepsilon_{M}=\alpha \cdot \frac{Q_{e}}{\sqrt{2 \pi} \sigma_{e}} \exp \left\{-\frac{\left[M \cdot v_{e}-\operatorname{Round}\left(M \cdot v_{e}\right)\right]^{2} \Delta T_{e}^{2}}{2 \sigma_{e}^{2}}\right\} .
$$

The coefficient $\alpha$ can be derived as (See APPENDIX),

$$
\alpha=\frac{Z_{i} e^{2} L_{\text {cool }}}{2 \varepsilon_{0} m_{i} \gamma^{3} \omega_{x} \pi a_{e}^{2}(\beta c)^{2}}=\frac{2 r_{0} \beta_{\text {avg }}}{\gamma^{3} \beta^{2} a_{e}^{2}} \frac{L_{\text {cool }}}{\beta c e},
$$

where

$$
\beta_{\text {avg }}=\frac{\beta c}{\omega_{x}},
$$

and

$$
r_{0}=\frac{Z_{i} e^{2}}{4 \pi \varepsilon_{0} m_{i} c^{2}} .
$$

Inserting eq. (9) into eq. (8) leads to

$$
\varepsilon_{M}=\frac{2 r_{0} \beta_{\text {avg }}}{\gamma^{3} \beta^{2} a_{e}^{2}} \frac{L_{\text {cool }}}{\beta c e} \cdot \frac{Q_{e}}{\sqrt{2 \pi} \sigma_{e}} \exp \left\{-\frac{\left[M \cdot v_{e}-\operatorname{Round}\left(M \cdot v_{e}\right)\right]^{2} \Delta T_{e}^{2}}{2 \sigma_{e}^{2}}\right\} .
$$

Applying eq. (77) to eq. (12), we obtain

$$
\begin{aligned}
\varepsilon_{M} & =4 \pi \cdot \Delta v_{e, M} \\
& =4 \pi \cdot \Delta v_{\text {peak }} \cdot \exp \left\{-\frac{\left[M \cdot v_{e}-\operatorname{Round}\left(M \cdot v_{e}\right)\right]^{2} \Delta T_{e}^{2}}{2 \sigma_{e}^{2}}\right\}
\end{aligned}
$$

with $v_{e}$ is the fractional part of $f_{e, \text { rep }} / f_{\text {rev }}$ and

$$
\Delta \nu_{\text {peak }}=\alpha \cdot \frac{Q_{e}}{4 \pi \sqrt{2 \pi} \sigma_{e}}=\frac{N_{e}}{2 \pi \sqrt{2 \pi} \sigma_{e}} \frac{r_{0} \beta_{\text {avg }}}{\gamma^{3} \beta^{3} a_{e}^{2}} \frac{L_{\text {cool }}}{c} .
$$

Using eq. (9) from [1], we have 


$$
\frac{J(N, \hat{\psi})}{J(0)}=\exp [u(N, \hat{\psi})]
$$

with

$$
\begin{gathered}
u(N, \hat{\psi})=-\sum_{k=0}^{N} \sin (4 \pi k \tilde{Q}+2 \hat{\psi}) \delta \varepsilon_{k}+\frac{1}{2}\left[\sum_{k=0}^{N} \cos (4 \pi k \tilde{Q}+2 \hat{\psi}) \delta \varepsilon_{k}\right]^{2}, \\
\varepsilon_{\text {avg }}=\frac{1}{N} \sum_{k=1}^{N} \varepsilon_{k} \\
\tilde{Q}=Q+\frac{\varepsilon_{a v g}}{4 \pi},
\end{gathered}
$$

and

$$
\delta \varepsilon_{k}=\varepsilon_{k}-\varepsilon_{\text {avg }} .
$$

The evolution of the phase is given by

$$
\begin{aligned}
\psi_{N} & =2 \pi \tilde{Q} N+\hat{\psi}+\sum_{i=0}^{N} \sin ^{2} \psi_{i} \delta \varepsilon_{i} \\
& \approx 2 \pi \tilde{Q} N+\hat{\psi}-\frac{1}{2} \sum_{i=0}^{N} \cos [4 \pi \tilde{Q} i+2 \hat{\psi}] \delta \varepsilon_{i}
\end{aligned}
$$

\section{Fourier Analysis}

In order to find out the resonant condition for emittance growth, we Fourier decompose eq. (1) as

$$
\begin{aligned}
I_{e}(t) & =\frac{Q_{e}}{\sqrt{2 \pi} \sigma_{e}} \sum_{n=-\infty}^{\infty} \exp \left[-\frac{\left(t-n \cdot \Delta T_{e}\right)^{2}}{2 \sigma_{e}^{2}}\right] \\
& =\frac{Q_{e}}{\sqrt{2 \pi} \sigma_{e}}\left\{\frac{a_{0}}{2}+\sum_{k=1}^{\infty}\left[a_{k} \cos \left(\frac{2 k \pi t}{\Delta T_{e}}\right)+b_{k} \sin \left(\frac{2 k \pi t}{\Delta T_{e}}\right)\right]\right\}^{\prime}
\end{aligned}
$$

with

$$
a_{k} \approx \frac{2 \sqrt{2 \pi} \sigma_{e}}{\Delta T_{e}} \exp \left(-\frac{2 k^{2} \pi^{2} \sigma_{e}^{2}}{\Delta T_{e}^{2}}\right),
$$

and

$$
b_{k} \approx 0 .
$$

for $\sigma_{e} \ll \Delta T_{e}$. Inserting eq. (22) and (23) into eq. (21) leads to

$$
\begin{aligned}
I_{e}(t) & =\frac{Q_{e}}{\sqrt{2 \pi} \sigma_{e}} \sum_{n=-\infty}^{\infty} \exp \left[-\frac{\left(t-n \cdot \Delta T_{e}\right)^{2}}{2 \sigma_{e}^{2}}\right] \\
& =\frac{2 Q_{e}}{\Delta T_{e}}\left\{\frac{1}{2}+\sum_{k=1}^{\infty} \exp \left(-\frac{2 k^{2} \pi^{2} \sigma_{e}^{2}}{\Delta T_{e}^{2}}\right) \cos \left(\frac{2 k \pi t}{\Delta T_{e}}\right)\right\}
\end{aligned}
$$


The average electron current seen by ions is thus

$$
\left\langle I_{e}\right\rangle_{\text {avg }}=\frac{2 Q_{e}}{\Delta T_{e}}\left\{\frac{1}{2}+\sum_{k=1}^{\infty} \Theta\left(N, v_{e}\right) \exp \left(-\frac{2 k^{2} \pi^{2} \sigma_{e}^{2}}{\Delta T_{e}^{2}}\right)\right\},
$$

with

$$
\Theta\left(N, v_{e}\right) \equiv \frac{1}{2(N+1)}\left\{1+\frac{\sin \left[(2 N+1) k \pi v_{e}\right]}{\sin \left(k \pi v_{e}\right)}\right\} .
$$

Inserting eq. (24) and (25) into eq. (5) and (19) yields

$$
\delta \varepsilon_{l}=\frac{2 \alpha Q_{e}}{\Delta T_{e}} \sum_{k=1}^{\infty} \exp \left(-\frac{2 k^{2} \pi^{2} \sigma_{e}^{2}}{\Delta T_{e}^{2}}\right) \cdot\left[\cos \left(\frac{2 k \pi l T_{r e v}}{\Delta T_{e}}\right)-\Theta\left(N, v_{e}\right)\right] .
$$

Applying eq. (27) to the first term of eq. (16) produces

$$
\begin{gathered}
u_{1}(N, \hat{\psi})=-\frac{4(2 \pi)^{\frac{3}{2}} \sigma_{e} \Delta v_{\text {peak }}}{\Delta T_{e}} \sum_{l=0}^{N} \sin (4 \pi l \tilde{Q}+2 \hat{\psi}) \sum_{k=1}^{\infty} \mathrm{e}^{-\frac{2 k^{2} \pi^{2} \sigma_{e}^{2}}{\Delta T_{e}^{2}}} \cdot\left[\cos \left(\frac{2 k \pi l T_{\text {rev }}}{\Delta T_{e}}\right)-\Theta\left(N, v_{e}\right)\right] \\
=-\frac{2(2 \pi)^{\frac{3}{2}} \sigma_{e} \Delta v_{\text {peak }}}{\Delta T_{e}}\left\{-2 \sum_{k=1}^{\infty} \mathrm{e}^{-\frac{2 k^{2} \pi^{2} \sigma_{e}^{2}}{\Delta T_{e}^{2}}} \Theta\left(N, v_{e}\right) \sum_{l=0}^{N} \sin (4 \pi I \tilde{Q}+2 \hat{\psi})+\right. \\
\left.\sum_{l=0}^{N} \sum_{k=1}^{\infty} \mathrm{e}^{-\frac{2 k^{2} \pi^{2} \sigma_{e}^{2}}{\Delta T_{e}^{2}}} \sin \left[2 \pi l\left(2 \tilde{Q}+\frac{k T_{\text {rev }}}{\Delta T_{e}}\right)+2 \hat{\psi}\right]+\sum_{l=0}^{N} \sum_{k=1}^{\infty} \mathrm{e}^{-\frac{2 k^{2} \pi^{2} \sigma_{e}^{2}}{\Delta T_{e}^{2}}} \sin \left[2 \pi l\left(2 \tilde{Q}-\frac{k T_{\text {rev }}}{\Delta T_{e}}\right)+2 \hat{\psi}\right]\right\}
\end{gathered}
$$

The second term of eq. (16) reads

$$
\begin{aligned}
u_{2}(N, \hat{\psi})= & \frac{1}{2}\left[\sum_{m=0}^{N} \cos (4 \pi m \tilde{Q}+2 \hat{\psi}) \delta \varepsilon_{m}\right]^{2} \\
& =64 \pi^{3}\left(\frac{\sigma_{e} \Delta v_{\text {peak }}}{\Delta T_{e}}\right)^{2} \sum_{l, k=1}^{\infty} \mathrm{e}^{-\frac{2\left(k^{2}+l^{2}\right) \pi^{2} \sigma_{e}^{2}}{\Delta T_{e}^{2}}} \sum_{m, n=0}^{N}\left[\cos \left(\frac{2 k \pi m T_{\text {rev }}}{\Delta T_{e}}\right)-\Theta\left(N, k v_{e}\right)\right] . \\
& {\left[\cos \left(\frac{2 l \pi n T_{\text {rev }}}{\Delta T_{e}}\right)-\Theta\left(N, l v_{e}\right)\right] \cos (4 \pi n \tilde{Q}+2 \hat{\psi}) \cos (4 \pi m \tilde{Q}+2 \hat{\psi}) }
\end{aligned}
$$

For $v_{e} \neq 0$, taking the leading term of $1 / N$ in eq. (29) leads to

$$
\begin{gathered}
u_{2}(N, \hat{\psi}) \approx 8 \pi^{3}\left(\frac{\sigma_{e} \Delta v_{\text {peak }}}{\Delta T_{e}}\right)^{2} \sum_{k=1}^{\infty} \mathrm{e}^{-\frac{4 k^{2} \pi^{2} \sigma_{e}^{2}}{\Delta T_{e}^{2}}} \sum_{m, n=0}^{N} \\
\left.\left\{\cos \left[2 \pi(n+m) \cdot\left(2 \tilde{Q}+\frac{T_{r e v}}{\Delta T_{e}} k\right)+4 \hat{\psi}\right]+\cos \left[2 \pi(n+m) \cdot\left(2 \tilde{Q}-\frac{T_{r e v}}{\Delta T_{e}} k\right)+4 \hat{\psi}\right] \cdot(30) \cdot\left(2 \tilde{Q}-\frac{T_{r e v}}{\Delta T_{e}} k\right)\right]\right\} \\
+\cos \left[2 \pi(n-m) \cdot\left(2 \tilde{Q}+\frac{T_{r e v}}{\Delta T_{e}} k\right)\right]+\cos [2 \pi(n-m)]
\end{gathered}
$$

Hence the resonant condition is 


$$
2 \tilde{Q} \pm \frac{k T_{r e v}}{\Delta T_{e}}=K,
$$

with $K$ being an integer. Letting $2 Q_{\text {dec }}$ be the fractional part of $2 \tilde{Q}$ and $v_{e}$ be the fractional part of $\frac{f_{\text {rep }}}{f_{\text {rev }}}$, eq. (31) becomes

$$
2 Q_{\text {dec }}=K \pm k v_{e} .
$$

If $2 \tilde{Q}-\frac{T_{r e v}}{\Delta T_{e}} k=0$, eq. (28) becomes

$$
u_{1}(N, \hat{\psi})=-\frac{2(2 \pi)^{\frac{3}{2}} \sigma_{e} \Delta v_{\text {peak }}}{\Delta T_{e}} N \mathrm{e}^{-\frac{2 k^{2} \pi^{2} \sigma_{e}^{2}}{\Delta T_{e}^{2}}} \sin (2 \hat{\psi})
$$

eq. (29) becomes

$$
u_{2}(N, \hat{\psi}) \approx 8 \pi^{3}\left(\frac{\sigma_{e} \Delta v_{\text {peak }}}{\Delta T_{e}}\right)^{2} N^{2} \mathrm{e}^{-\frac{4 k^{2} \pi^{2} \sigma_{e}^{2}}{\Delta T_{e}^{2}}}[1+\cos (4 \hat{\psi})]
$$

Inserting eq. (33) and (34) into eq. (15) yields

$$
\frac{J(N, \hat{\psi})}{J(0)}=\exp \left[u_{k}(N, \hat{\psi})\right]
$$

where

$$
u_{k}(N, \hat{\psi})=-2 g_{k} N \sin (2 \hat{\psi})+g_{k}^{2} N^{2}[1+\cos (4 \hat{\psi})]
$$

and

$$
g_{k} \equiv(2 \pi)^{\frac{3}{2}} \frac{\sigma_{e} \Delta v_{\text {peak }}}{\Delta T_{e}} \mathrm{e}^{-\frac{2 k^{2} \pi^{2} \sigma_{e}^{2}}{\Delta T_{e}^{2}}} .
$$

Inserting eq. (36) into eq. (35) and averaging over the initial phase yields

$$
\begin{aligned}
J_{\text {avg }}(N) & =J(0) \frac{1}{2 \pi} \int_{0}^{2 \pi} \exp \left[u_{k}(N, \hat{\psi})\right] d \hat{\psi} \\
& =J(0) \frac{1}{2 \pi} \int_{0}^{2 \pi} \exp \left\{-2 g_{k} N \sin (2 \hat{\psi})+g_{k}{ }^{2} N^{2}[1+\cos (4 \hat{\psi})]\right\} d \hat{\psi}
\end{aligned}
$$

For $u_{k}(N, \hat{\psi})<<1$, eq. (38) becomes

$$
\begin{aligned}
J_{\text {avg }}(N) & =J(0) \frac{1}{2 \pi} \int_{0}^{2 \pi} \exp \left\{-2 g_{k} N \sin (2 \hat{\psi})+g_{k}^{2} N^{2}[1+\cos (4 \hat{\psi})]\right\} d \hat{\psi} \\
& \approx \frac{J(0)}{2 \pi} \int_{0}^{2 \pi}\left\{1+g_{k}^{2} N^{2}+2 g_{k}^{2} N^{2} \sin ^{2}(2 \hat{\psi})\right\} d \hat{\psi} \\
& =J(0)\left(1+2 g_{k}{ }^{2} N^{2}\right)
\end{aligned} .
$$




\section{Simple Tracking}

Using the normalized canonical variables:

$$
P_{x}=\frac{p_{x}}{\sqrt{Q_{x}}}=\sqrt{2 J} \cos \psi
$$

and

$$
X=x \sqrt{Q_{x}}=\sqrt{2 J} \sin \psi,
$$

the one turn transfer map can be written as

$$
X_{n}(k+1)=\cos \left(2 \pi Q_{x}\right) X_{n}(k)+\sin \left(2 \pi Q_{x}\right) P_{n}(k)
$$

and

$$
P_{n}(k+1)=\cos \left(2 \pi Q_{x}\right) P_{n}(k)-\sin \left(2 \pi Q_{x}\right) X_{n}(k)+\varepsilon_{k} X_{n}(k+1) .
$$

The tracking is done in Mathcad and the results are shown in fig. 7 for the relative growth of the average action growth of 300 macro-particles with the same initial action and evenly uniformly distributed phases. The betatron tune is taken as $Q_{x}=28.15$ and the electron bunch repetition frequency is taken such that the fractional part of $f_{e, \text { rep }} / f_{\text {rev }}$ is 0.3 . As shown in fig. 3 , the square growth formula, eq. (39) fully agrees with the tracking results. Fig. 8 shows the phase space plot comparison between the initial and final distribution after 2000 turns.

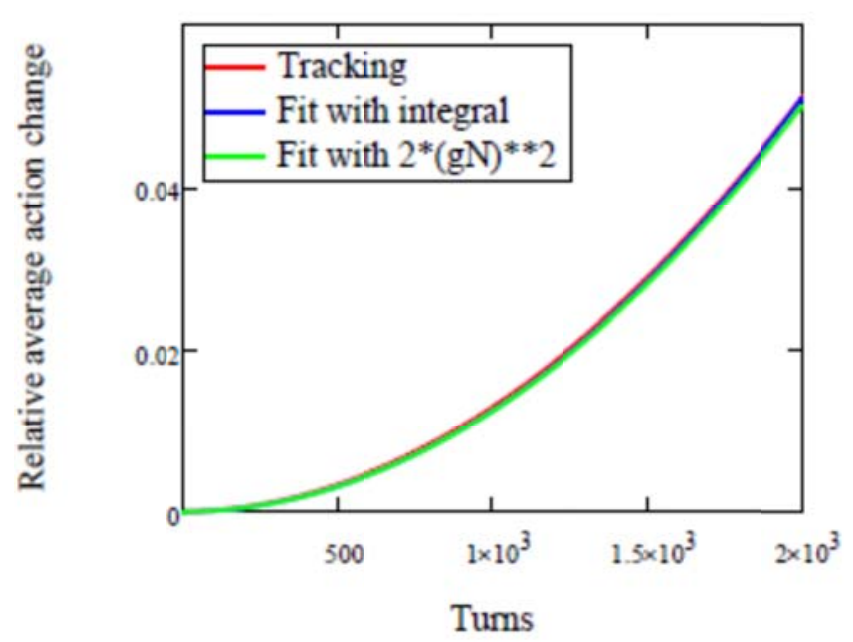

Figure 3: comparison of average action growth as calculated from eq. (38) and (39) with direct time domain tracking according to eq. (40) and (43). 


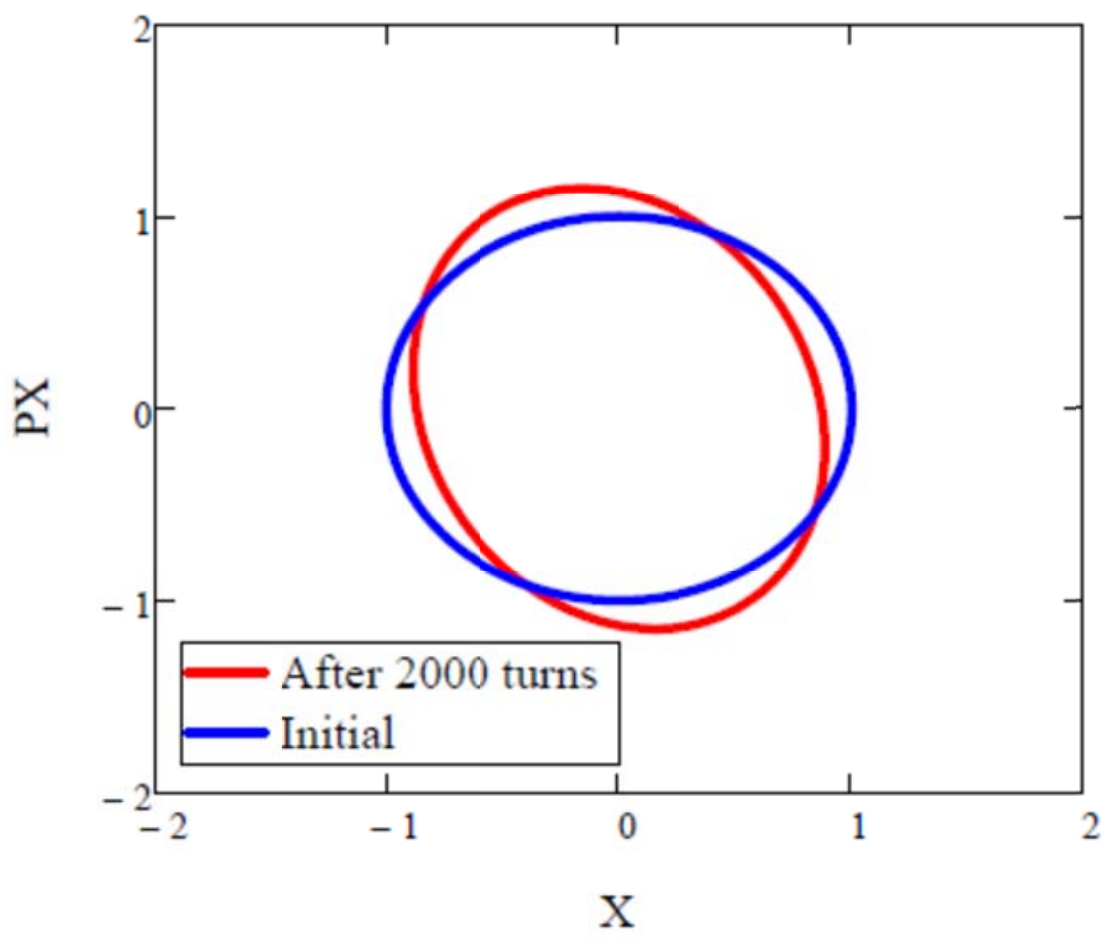

Figure 4: comparison of phase space distribution. The blue curve is the initial phase space distribution in normalized variables and the red curve is the phase space distribution after 2000 turns.

\section{Requirement on Electron Bunch Repetition Frequency Accuracy}

In order to store the ions for $N_{\text {store }}$ turns with the action growth below a factor of two, the highest order resonances to avoid is given by eq. (39), i.e.

$$
g_{k}=(2 \pi)^{\frac{3}{2}} \frac{\sigma_{e} \Delta v_{\text {peak }}}{\Delta T_{e}} \mathrm{e}^{-\frac{2 k^{2} \pi^{2} \sigma_{e}^{2}}{\Delta T_{e}^{2}}}<\frac{1}{\sqrt{2} N_{\text {store }}},
$$

which can be re-written as

$$
k^{2}>\frac{\Delta T_{e}^{2}}{2 \pi^{2} \sigma_{e}^{2}} \ln \left[\frac{\sqrt{2}(2 \pi)^{\frac{3}{2}} N_{\text {store }} \sigma_{e} \Delta v_{\text {peak }}}{\Delta T_{e}}\right] .
$$

Eq. (45) suggests that the growth will always slower than the requirement if the space charge tune shift is smaller than

$$
\Delta v_{\text {peak }}<\frac{\Delta T_{e}}{\sqrt{2}(2 \pi)^{\frac{3}{2}} N_{\text {store }} \sigma_{e}} .
$$

If eq. (46) is not satisfied, eq. (45) requires 


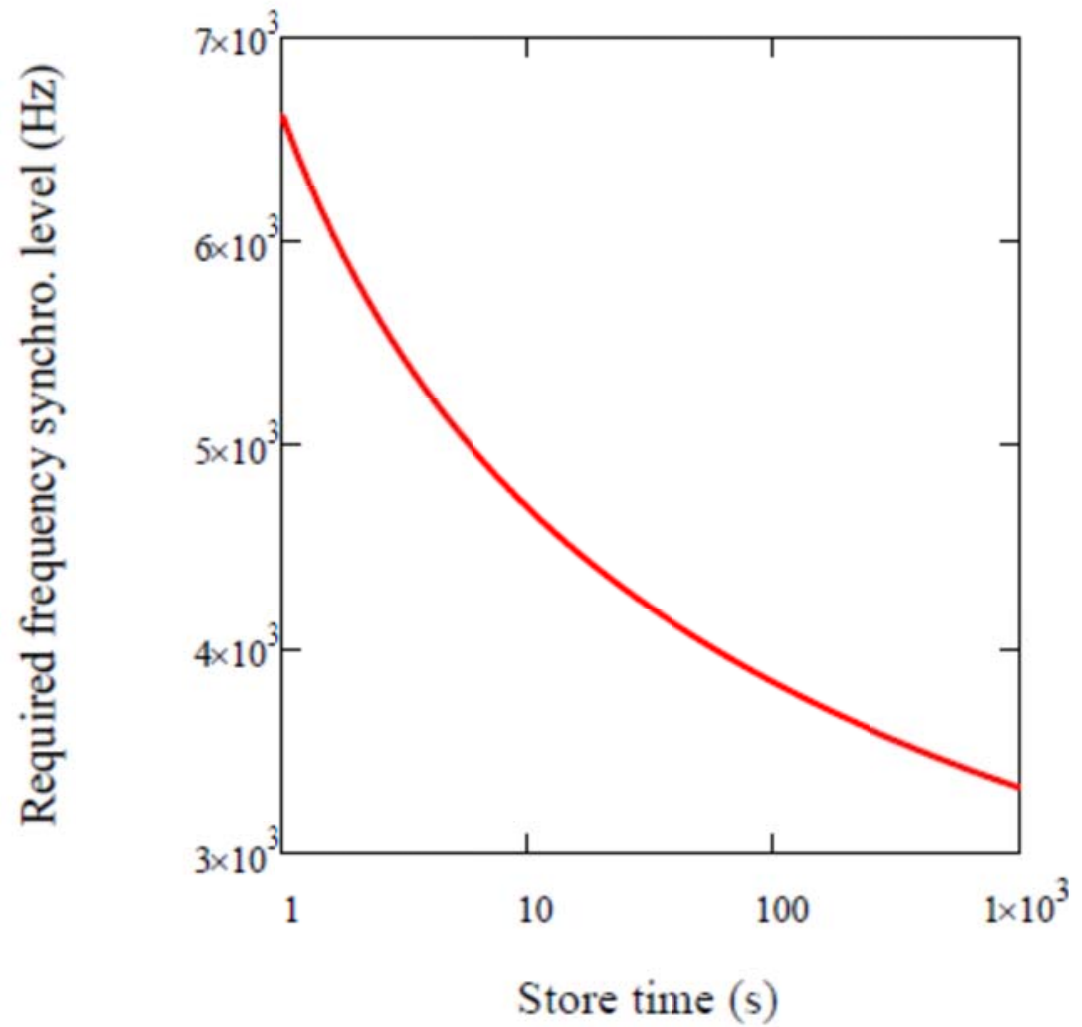

Figure 5: required electron repetition frequency accuracy level as a function of ion beam store time. The plot is generated from eq. (49) for $\Delta T_{e}=10 \mathrm{~ns}, \sigma_{e}=1 \mathrm{~ns}$, $Q_{e}=4 n C, \Delta v_{\text {peak }}=3 \times 10^{-4}$ and $\gamma=4.1$.

$$
k>\frac{\Delta T_{e}}{\sqrt{2} \pi \sigma_{e}} \sqrt{\ln \left[\frac{\sqrt{2}(2 \pi)^{\frac{3}{2}} N_{\text {store }} \sigma_{e} \Delta v_{\text {peak }}}{\Delta \boldsymbol{T}_{e}}\right]} .
$$

Assuming $v_{e}$ is close to zero, applying eq. (32) yields

$$
\frac{\min \left(2 \tilde{Q}_{\text {dec }}, 1-2 \tilde{Q}_{\text {dec }}\right)}{v_{e}}>\frac{\Delta T_{e}}{\sqrt{2} \pi \sigma_{e}} \sqrt{\ln \left[\frac{\sqrt{2}(2 \pi)^{\frac{3}{2}} N_{\text {store }} \sigma_{e} \Delta v_{\text {peak }}}{\Delta T_{e}}\right]},
$$

i.e.

$$
\delta f_{\text {rep }}<\sqrt{2} \pi \cdot \frac{\sigma_{e}}{\Delta T_{e}} \cdot f_{\text {rev }} \cdot \min \left(2 \tilde{Q}_{\text {dec }}, 1-2 \tilde{Q}_{\text {dec }}\right) \cdot\left\{\ln \left[\frac{\sqrt{2}(2 \pi)^{\frac{3}{2}} \sigma_{e} \Delta v_{\text {peak }} N_{\text {store }}}{\Delta T_{e}}\right]\right\}^{-\frac{1}{2}}
$$

Figure 5 shows the dependence of the required electron bunch repetition frequency as a function of RHIC ion beam storing time as calculated from eq. (49). For parameters listed in the caption of fig. 5, the required accuracy of the electron bunch repetition frequency is $3 \mathrm{KHz}$. 


\section{Estimation of Required Arriving Time Jitter Level}

In the presence of arriving time jitter, eq. (13) becomes

$$
\varepsilon_{M}=4 \pi \cdot \Delta v_{\text {peak }} \cdot \exp \left\{-\frac{\left[\tau+M \cdot v_{e} \Delta T_{e}-\operatorname{Round}\left(M \cdot v_{e}\right) \Delta T_{e}+\delta_{t, M}\right]^{2}}{2 \sigma_{e}^{2}}\right\},
$$

with $\delta_{t, M}$ being the arriving time error in the $M^{\text {th }}$ turn and $\tau$ is the location of the ion. If $v_{e}=0$, eq. (50) becomes

$$
\varepsilon_{M}=4 \pi \cdot \Delta v_{\text {peak }} \cdot \exp \left(-\frac{\left(\tau+\delta_{t, M}\right)^{2}}{2 \sigma_{e}^{2}}\right) .
$$

The maximal kick variation happens at

$$
\begin{aligned}
\frac{d^{2}}{d \tau^{2}} \varepsilon_{M} & =4 \pi \cdot \Delta v_{\text {peak }} \cdot \frac{d}{d \tau}\left[-\frac{\tau}{\sigma_{e}^{2}} \exp \left(-\frac{\tau^{2}}{2 \sigma_{e}^{2}}\right)\right] \\
& =4 \pi \cdot \Delta v_{\text {peak }} \cdot \frac{1}{\sigma_{e}^{2}} \exp \left(-\frac{\tau^{2}}{2 \sigma_{e}^{2}}\right)\left[\frac{\tau^{2}}{\sigma_{e}^{2}}-1\right], \\
& =0
\end{aligned}
$$

i.e.

$$
\tau_{0}=\sigma_{e} .
$$

If the arriving time jitter is much smaller than the bunch length, eq. (51) becomes

$$
\begin{aligned}
\varepsilon_{M} & =4 \pi \cdot \Delta v_{\text {peak }} \cdot \exp \left(-\frac{\left(1+\delta_{t, M} / \sigma_{e}\right)^{2}}{2}\right) \\
& \approx 4 \pi \cdot \Delta v_{\text {peak }} \cdot \exp \left(-\frac{\left(1+2 \delta_{t, M} / \sigma_{e}\right)}{2}\right), \\
& =\frac{4 \pi}{\sqrt{e}} \cdot \Delta v_{\text {peak }} \cdot \exp \left(-\frac{\delta_{t, M}}{\sigma_{e}}\right) \\
& \approx \frac{4 \pi}{\sqrt{e}} \cdot \Delta v_{\text {peak }} \cdot\left(1-\frac{\delta_{t, M}}{\sigma_{e}}\right)
\end{aligned}
$$

which leads to the average kick of

$$
\frac{1}{N} \sum_{i=1}^{N} \varepsilon_{i} \approx \frac{4 \pi}{\sqrt{e}} \cdot \Delta v_{\text {peak }},
$$

with the rms variation of 


$$
\begin{aligned}
\left\langle\delta \varepsilon^{2}\right\rangle & =\frac{1}{N} \sum_{i=1}^{N}\left(\varepsilon_{i}-\langle\varepsilon\rangle\right)^{2} \\
& \approx \frac{1}{N} \sum_{i=1}^{N}\left(\frac{4 \pi}{\sigma_{e} \sqrt{e}} \cdot \Delta v_{\text {peak }} \cdot \delta_{t, M}\right)^{2} \\
& =\left(\frac{4 \pi \Delta v_{\text {peak }}}{\sigma_{e} \sqrt{e}}\right)^{2} \frac{1}{N} \sum_{i=1}^{N}\left(\delta_{t, M}\right)^{2} \\
& =\left(\frac{4 \pi \Delta v_{\text {peak }}}{\sigma_{e} \sqrt{e}}\right)^{2} \cdot\left\langle\delta_{t}^{2}\right\rangle
\end{aligned}
$$

Eq. (13) of [1] reads

$$
\ln \left(\frac{J_{N}}{J_{0}}\right)=N \frac{\left\langle\delta \varepsilon^{2}\right\rangle}{2} \frac{1-e^{-2 \alpha}}{1+e^{-2 \alpha}-2 e^{-\alpha} \cos (4 \pi Q)},
$$

with $\alpha$ being the characteristic parameter of the time correlation of the jitter, i.e.

$$
\left\langle\delta \varepsilon_{k} \delta \varepsilon_{m+k}\right\rangle=\left\langle\delta \varepsilon^{2}\right\rangle \exp (-\alpha|m|) .
$$

Assuming $\alpha \rightarrow \infty$, i.e. there is no correlation between arriving time jitters for two different turns, eq. (57) reduces to

$$
\ln \left(\frac{J_{N}}{J_{0}}\right)=N \frac{\left\langle\delta \varepsilon^{2}\right\rangle}{2} .
$$

Inserting eq. (56) into eq. (59) yields

$$
\begin{aligned}
J_{N} & =J_{0} \exp \left\{\frac{N}{2}\left(\frac{4 \pi \Delta v_{\text {peak }}}{\sigma_{e} \sqrt{e}}\right)^{2} \cdot\left\langle\delta_{t}^{2}\right\rangle\right\} \\
& \equiv J_{0} \exp \left(\frac{t}{\tau_{\text {grow }}}\right)
\end{aligned}
$$

with the growing time of

$$
\tau_{\text {grow }}=\frac{e}{8 \pi^{2}} T_{\text {rev }} \frac{\sigma_{e}^{2}}{\left\langle\delta_{t}^{2}\right\rangle} \frac{1}{\Delta v_{\text {peak }}^{2}} .
$$

Requiring the growing time to be longer than the store time leads to

$$
\sqrt{\left\langle\delta_{t}^{2}\right\rangle}<\frac{1}{2 \pi} \frac{\sigma_{e}}{\Delta v_{\text {peak }}} \sqrt{\frac{e}{2 N_{\text {store }}}} .
$$

Fig. 6 shows the required arriving time jitter level as a function of the beam store time. For the action growth below a factor of e, the required arrival time jitter is 37 ps. 


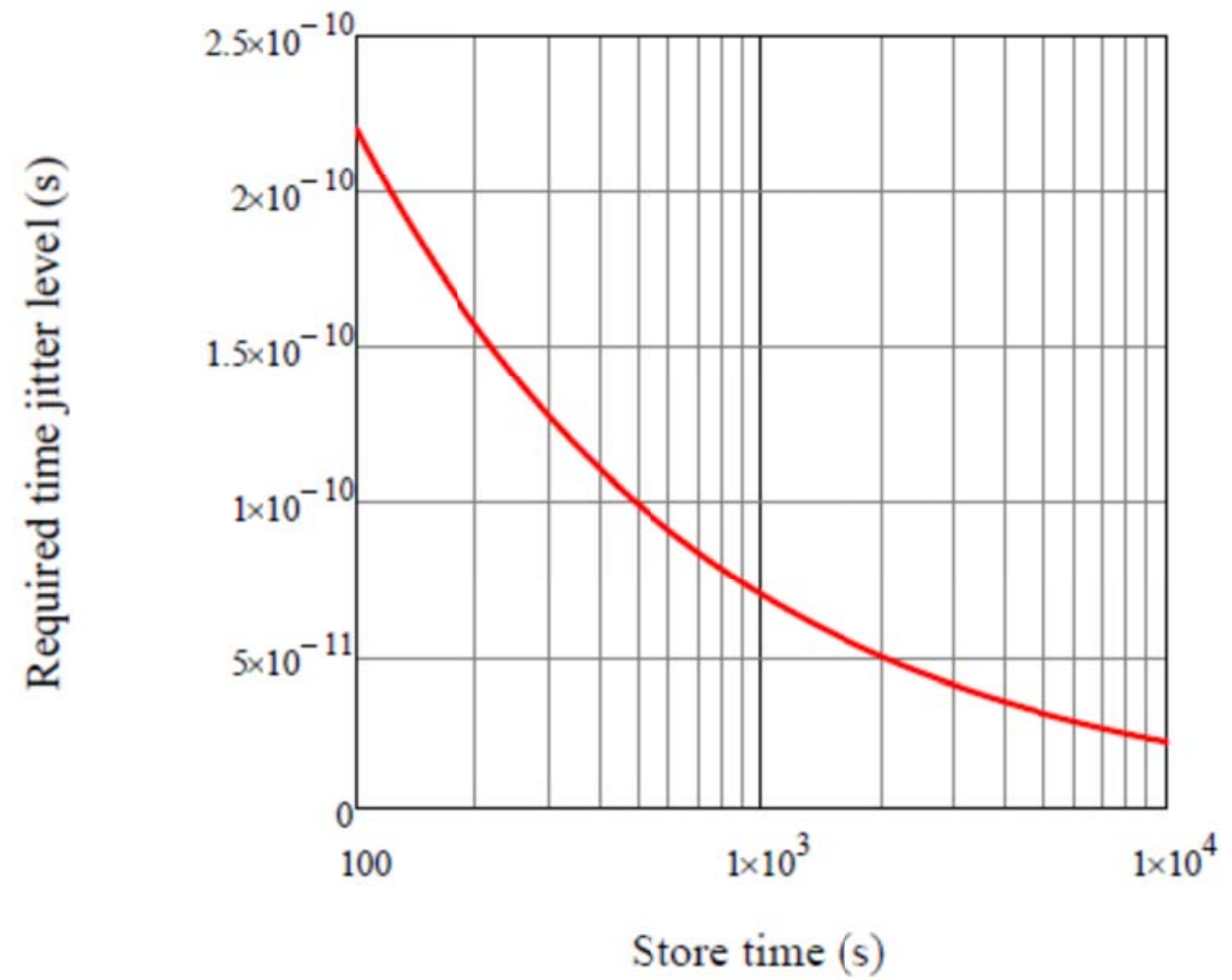

Figure 6: required time jitter level as calculated from eq. (62). It is assumed that there is no correlation between time jitters of two different turns.

\section{Estimation of Required Peak Current Jitter Level}

The maximal focusing strength is at the electron bunch center and is related to the electron bunch peak current by

$$
\varepsilon_{M}=\alpha \cdot I_{e}(M, 0),
$$

with $\alpha$ defined in eq. (9). The rms variation of the focusing strength is thus

$$
\left\langle\delta \varepsilon^{2}\right\rangle=\frac{1}{N} \sum_{i=1}^{N}\left(\varepsilon_{i}-\langle\varepsilon\rangle\right)^{2}=\alpha^{2} \cdot \frac{1}{N} \sum_{i=1}^{N}\left(I_{e}(i, 0)-\left\langle I_{e}(0)\right\rangle\right)^{2}=\alpha^{2}\left\langle\delta I_{e}(0)^{2}\right\rangle .
$$

According to eq. (14), the coefficient $\alpha$ can be written as

$$
\alpha=4 \pi \frac{\left\langle\Delta v_{\text {peak }}\right\rangle}{\left\langle I_{e}(0)\right\rangle} .
$$

Inserting eq. (65) into (64) produces

$$
\left\langle\delta \varepsilon^{2}\right\rangle=16 \pi^{2}\left\langle\Delta v_{\text {peak }}\right\rangle^{2} \frac{\left\langle\delta I_{e}(0)^{2}\right\rangle}{\left\langle I_{e}(0)\right\rangle^{2}},
$$

which combines with eq. (57) leads to 


$$
\ln \left(\frac{J_{N}}{J_{0}}\right)=8 \pi^{2} N\left\langle\Delta v_{\text {peak }}\right\rangle^{2} \frac{\left\langle\delta I_{e}(0)^{2}\right\rangle}{\left\langle I_{e}(0)\right\rangle^{2}} \frac{1-e^{-2 \alpha}}{1+e^{-2 \alpha}-2 e^{-\alpha} \cos (4 \pi Q)} .
$$

Assuming peak current jitters from two different turns are uncorrelated, i.e. $\alpha \rightarrow \infty$, eq. (67) reduces to

$$
\ln \left(\frac{J_{N}}{J_{0}}\right)=8 \pi^{2} N\left\langle\Delta v_{\text {peak }}\right\rangle^{2} \frac{\left\langle\delta I_{e}(0)^{2}\right\rangle}{\left\langle I_{e}(0)\right\rangle^{2}},
$$

which can be rewritten as

$$
J_{N}=J_{0} \exp \left\{8 \pi^{2} N\left\langle\Delta v_{\text {peak }}\right\rangle^{2} \frac{\left\langle\delta I_{e}(0)^{2}\right\rangle}{\left\langle I_{e}(0)\right\rangle^{2}}\right\} \equiv J_{0} \exp \left(\frac{N}{N_{\text {grow }}}\right),
$$

with

$$
N_{\text {grow }}=\frac{1}{8 \pi^{2}\left\langle\Delta v_{\text {peak }}\right\rangle^{2} \cdot \frac{\left\langle\delta I_{e}(0)^{2}\right\rangle}{\left\langle I_{e}(0)\right\rangle^{2}}} .
$$

Hence requiring the action growth time longer than the beam store time (or cooling time) gives the requirement for the current jitter as

$$
\frac{\sqrt{\left\langle\delta I_{e}(0)^{2}\right\rangle}}{\left\langle I_{e}(0)\right\rangle}<\frac{1}{2 \pi\left\langle\Delta v_{\text {peak }}\right\rangle} \sqrt{\frac{T_{\text {rev }}}{2 t_{\text {store }}}} .
$$

Fig.7 plots the required peak current jitter level as calculated from eq. (71) with parameters given in the caption of fig. 5 . As shown in fig. 7 , in order to have the local growth time smaller than 1000 seconds, the required peak current jitter level is below $4.3 \%$. 


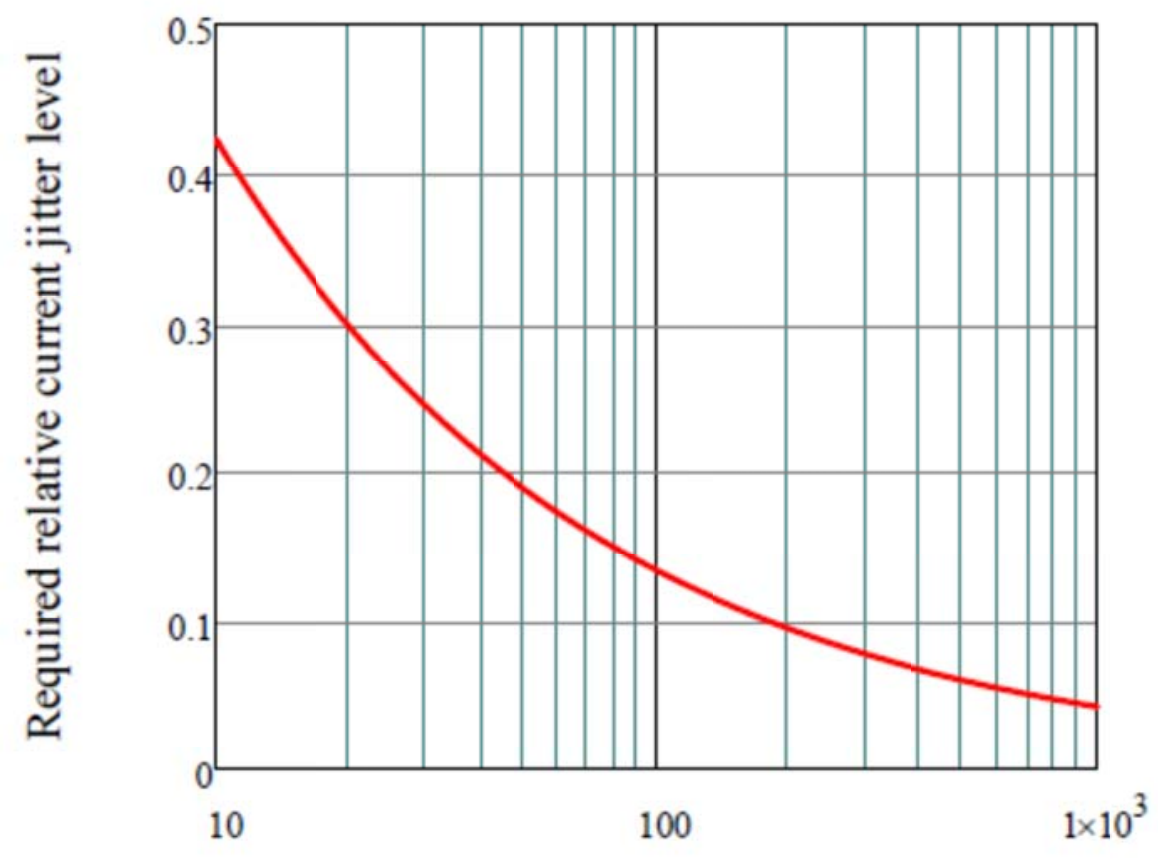

Store time (s)

Figure 7: required relative peak current jitter level as calculated from eq. (71). The jitters from two different turns are assumed to be uncorrelated. The parameters applied are given in the caption of fig. 5 .

\section{APPENDIX A:}

Now we will find the coefficient $\alpha$ so that $\delta \varepsilon_{M}$ has the same definition of reference [1]. According to eq. (1) of [1], the equation of motion reads

$$
\frac{d^{2}}{d \theta^{2}} x=-Q^{2} x-\delta_{p}(\theta) Q_{x} \varepsilon_{M}(\theta),
$$

with

$$
\theta=2 \pi t / T_{\text {rev }}
$$

Inserting eq. (73) into eq. (72) leads to

$$
\begin{aligned}
\frac{d^{2}}{d t^{2}} x & =-\frac{(2 \pi)^{2}}{T_{\text {rev }}^{2}} Q^{2} x-\frac{\delta_{p}(t)}{d \theta / d t} \frac{(2 \pi)^{2}}{T_{r e v}^{2}} Q \varepsilon_{M}(t) x \\
& =-\frac{(2 \pi)^{2}}{T_{r e v}^{2}} Q^{2} x-\delta_{p}(t) \frac{(2 \pi)}{T_{r e v}} Q \varepsilon_{M}(t) x \\
& =-\omega_{x}^{2} x-\delta_{p}(t) \omega_{x} \varepsilon_{M}(t) x
\end{aligned}
$$


Taking

$$
x=A \cos \left[\left(Q+\Delta v_{e}\right) \omega_{r e v} t\right]
$$

and inserting it into eq. (74) leads to

$$
\left(Q+\Delta v_{e}(t)\right)^{2}=Q^{2}+\delta_{p}(t) \frac{T_{r e v}}{2 \pi} Q \varepsilon_{M}(t)
$$

Integrating eq. (76) for the $M^{\text {th }}$ turn leads to

$$
\begin{aligned}
& 2 \int_{(M-1) T_{\text {rev }}}^{M T_{\text {rev }}} \Delta v_{e}(t) d t=\frac{T_{\text {rev }}}{2 \pi} \varepsilon_{M}(0) \\
& \Rightarrow \Delta v_{e, M}=\frac{1}{T_{\text {rev }}} \int_{(M-1) T_{\text {rev }}}^{M T_{\text {rev }}} \Delta v_{e}(t) d t=\frac{1}{4 \pi} \varepsilon_{M}(0)
\end{aligned} .
$$

The space charge field from the electron beam in the co-moving frame is

$$
\begin{gathered}
\vec{E}_{\perp}{ }^{\prime}=\frac{e n_{e}{ }^{\prime}}{2 \varepsilon_{0}} \vec{r}_{\perp}{ }^{\prime}, \\
E_{z}{ }^{\prime}=0,
\end{gathered}
$$

and

Explicitly, eq. (78) can be written as

$$
\vec{B}^{\prime}=0
$$

$$
E_{x}^{\prime}=\frac{-e n_{e}{ }^{\prime}}{2 \varepsilon_{0}} x^{\prime}
$$

and

$$
E_{y}^{\prime}=\frac{-e n_{e}{ }^{\prime}}{2 \varepsilon_{0}} y^{\prime} .
$$

In lab frame, the field is Lorentz transformed to

$$
\begin{gathered}
E_{x}=\frac{-e n_{e}}{2 \varepsilon_{0}} x, \\
E_{y}=\frac{-e n_{e}}{2 \varepsilon_{0}} y, \\
B_{x}=-\gamma \beta \frac{E_{y}{ }^{\prime}}{c}=\beta \frac{e n_{e}}{2 \varepsilon_{0} c} y,
\end{gathered}
$$

and

$$
B_{y}=\gamma \beta \frac{E_{x}^{\prime}}{c}=-\beta \frac{e n_{e}}{2 \varepsilon_{0} c} x .
$$

Thus the space charge force that the ions experienced from the electrons is

and

$$
F_{x}=Z_{i} e\left(E_{x}-\beta c B_{y}\right)=-\frac{Z_{i} e^{2} n_{e}}{2 \varepsilon_{0} \gamma^{2}} x,
$$




$$
F_{y}=Z_{i} e\left(E_{y}+\beta c B_{x}\right)=-\frac{Z_{i} e^{2} n_{e}}{2 \varepsilon_{0} \gamma^{2}} y .
$$

The equation of motion is thus

$$
\frac{d^{2}}{d t^{2}} x=-\omega_{x}^{2} x+\frac{1}{m_{i} \gamma} F_{x}=-\omega_{x}^{2} x-\frac{Z_{i} e^{2} n_{e} L_{c o o l}}{2 \varepsilon_{0} m_{i} \gamma^{3} \beta c} \delta_{p}(t) x .
$$

Comparing eq. (89) with eq. (74) yields

$$
\varepsilon_{M}(t)=\frac{Z_{i} e^{2} n_{e}(t)}{2 \varepsilon_{0} m_{i} \gamma^{3} \omega_{x}}=\frac{Z_{i} e^{2} L_{\text {cool }} I_{e}(t)}{2 \varepsilon_{0} m_{i} \gamma^{3} \omega_{x} \pi a_{e}^{2}(\beta c)^{2} e} .
$$

Comparing eq. (90) with eq. (5) leads to

$$
\alpha=\frac{Z_{i} e^{2} L_{\text {cool }}}{2 \varepsilon_{0} m_{i} \gamma^{3} \omega_{x} \pi a_{e}^{2}(\beta c)^{2}}=\frac{2 r_{0} \beta_{\text {avg }}}{\gamma^{3} \beta^{2} a_{e}^{2}} \frac{L_{\text {cool }}}{\beta c e},
$$

\section{References}

[1] M. Blaskiewicz, in C-A/AP/\#363, 2009. 